DOI: 10.20472/IAC.2019.051.037

\title{
JINDAPORN SANGGANJANAVANISH
}

Suranaree Univeristy of Technology, Thailand

\section{INVESTIGATING THE RELATIONSHIP OF ANALYTIC SCORING CRITERIA TO THE HOLISTIC ASSESSMENT OF SPEAKING PERFORMANCE}

\begin{abstract}
:
Two approaches in assessing speaking ability are holistic scoring and analytic scoring. In the holistic approach, an examinee's speech is given a single rating to reflect its overall quality. Analytic scoring, on the other hand, involves different aspects of performance, with each receiving its own rating. The present study investigated the relationship of analytic scoring criteria to the holistic assessment of speaking performance. It aimed to discover whether raters, in assigning a single global rating, had a tendency to focus significantly more on certain aspects of speaking than others. Ten experienced raters judged speech performance of thirty-five non-native English speakers. Two types of scores were obtained - a single score for holistic rating and a set of scores corresponding to five analytic criteria, i.e. coherence, fluency, grammatical accuracy, lexical resource, and pronunciation. Multiple regression analysis revealed that fluency had the strongest impact on the holistic score, followed by pronunciation, coherence, and lexical resource, while grammatical accuracy did not contribute significantly to the rating. Despite the slight differences in the weight contribution of each criterion to the overall score, the findings suggest that raters seemed to favor the ability to communicate at the right speed without too many pronunciation difficulties, while focusing less on lexical and grammatical accuracy.
\end{abstract}

\section{Keywords:}

holistic scoring, analytic scoring, speaking assessment 